\title{
Häusliche Pflege jetzt auch im Heim verordnungsfähig
}

Eine Änderung in der aktuellen Fassung der Häuslichen Krankenpflege-Richtlinien hat dazu geführt, dass eine Verordnung von Behandlungspflege über Muster 12 auch für Versicherte in Pflegeheimen zulässig ist. Es muss dabei allerdings ein besonders hoher Bedarf an medizinischer Behandlungspflege für die Dauer von voraussichtlich mindestens sechs Monaten vorliegen. Diese Ausnahmeregelung besteht deshalb nur für ein ganz bestimmtes Patientenklientel. Dies ist der Fall, wenn aus medizinischen Gründen die ständige Anwesenheit einer geeigneten Pflegekraft zur individuellen Kontrolle und Einsatzbereitschaft oder ein vergleichbar intensiver Einsatz einer Pflegefachkraft erforderlich ist.

\section{MMW Kommentar}

In diese Rubrik fallen in erster Linie Patienten mit schwer beeinträchtigter oder fehlender Selbstversorgungsfähigkeit, schwer gestörter oder fehlender Mobilität, schwer beeinträchtigter oder fehlender Kommunika-

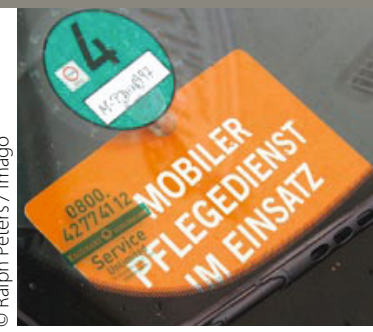

Hirnverletzungen, ein Zustand nach ischämischem Schlaganfall, Hirnblutungen, Sinusvenenthrombose, der Zustand nach entzündlichen Erkrankungen wie Enzephalitis, toxische Schädigungen, fortschreitende hirndegenetionsfähigkeit oder schweren Störungen im Verhalten, ggf. mit Abhängigkeit von lebenserhaltenden Hilfsmitteln. Pflegebegründende Diagnosen können Erkrankungen des ZNS sein, wie z.B. ein Zustand nach Sauerstoffmangelversorgung, ein apallisches Syndrom, traumatische rative Erkrankungen wie z.B. Chorea Huntington mit ausgeprägten neurologischen Funktionsstörungen im fortgeschrittenen Krankheitsverlauf oder Hirntumore. Darüber hinaus muss mindestens eine Pflegebedürftigkeit nach SGB XI im Sinne der Pflegestufe 2 vorliegen.

\section{Kostenerstattung statt „Aut idem“: Der Patient zahlt!}

— Mit der zum 1. Januar 2011 in Kraft getretenen Änderung des $\$ 129$ Abs. 1 SGB V wird die sog. Aut-idem-Substitution in Apotheken neu geregelt. Bei einer Wirkstoffverordnung oder einer Verordnung ohne Autidem-Kreuz auf dem Rezept müssen Apo- theken zukünftig ein preisgünstiges bzw. rabattiertes Arzneimittel abgeben, das mit dem verordneten in Wirkstärke und Packungsgröße identisch ist, für nur noch ein gleiches Anwendungsgebiet zugelassen ist und die gleiche oder eine austauschbare
Darreichungsform besitzt. Diese Neuregelung der Aut-idem-Substitution sorgt bei Herstellern und in Apotheken zur Zeit für große Verwirrung und führt in der Regel dazu, dass der Arzt aufgefordert wird, generell das Aut-idem-Kreuz zu setzen.

\section{MMW Kommentar}

Bedauerlicherweise bedienen sich mittlerweile auch Kassenangestellte dieser subtilen Methode, Ärztinnen und Ärzte in vermehrte Regressgefahr zu bringen. Eine Aut-idem-Substitution ist nämlich nur dann wirtschaftlich, wenn sie aus nachweislich individuell medizinischen Gründen notwendig ist. Fehlt eine solche Indikation, greift eine andere, seit dem 1.1.2011 gültige gesetzliche Regelung. Versicherte können nämlich in der Apotheke gegen Kostenerstattung ein anderes, nicht rabattiertes Arzneimittel (gleicher Wirkstoff, gleiche Wirkstärke, gleiche Packungsgröße und ein gleiches Anwendungsgebiet) statt des verordneten Arzneimittels erhalten, wobei die Versicherten die Differenz zwischen dem abgegebenen und dem rabattierten Präparat selbst zu tragen haben. Sie sind dabei nicht für ein Jahr an ihre Wahl der Kostenerstattung gebunden (\$13 Abs. 2 Satz 12 SGB V gilt hier nicht).

Die Krankenkasse darf in diesem Fall der Kostenerstattung auch keine Abschläge für fehlende Wirtschaftlichkeitsprüfungen vorsehen. Sie kann aber die Kosten für die entgangenen Rabatte nach §130a Abs. 8 SGB V sowie Mehrkosten im Vergleich zu einer Abgabe entsprechend der Aut-Idem-Regelung berücksichtigen. Das ist für die Kassenbürokraten natürlich lästig, denn sie müssen ihren Versicherten das Rabattsystem erläutern. Bei der Umsetzung dieser neuen Regelung bestehen deshalb noch viele Unklarheiten. Die Apotheke kann dem Patienten keine Auskunft darüber geben, welchen Betrag er von seiner Krankenkasse erstattet bekommt. Viele Krankenkassen können hierzu auch noch keine Aussage machen, obgleich sie bei Arzneimittel-Richtgrößenprüfungen so tun, als ob sie das könnten und Regressanträge stellen.

Für den Patienten kommt hinzu, dass die Erstattung bei der Krankenkasse nicht sofort erfolgt, sondern eine teilweise lange Bearbeitungszeit zur Folge hat. Dies alles führt dazu, dass letztendlich die Arztpraxis mit einem Thema konfrontiert wird, von dem sie eigentlich nicht betroffen ist und auf das sie keinerlei Einfluss hat. 